\title{
Oral Mucosal Grafts: Old Technique in New Light
}

\author{
Christian Mai Eckart Bertelmann \\ Augenklinik, Charité Campus Virchow-Klinikum, Berlin, Germany
}

\author{
Key Words \\ Mucous membrane graft · Fornix reconstruction . \\ Eyelid reconstruction - Conjunctival replacement
}

\begin{abstract}
Purpose: To present an update on indications and efficacy of oral mucosal grafts in the clinical management of ocular surface diseases focusing on the new developments of the last 5 years. Methods: Pubmed research on the databases of the years 2007 up to 2012 for the key words mucous membrane graft and eye, fornix reconstruction, eyelid reconstruction, and conjunctival replacement. Results: Well-documented clinical indications for oral mucosal grafting to the ocular surface include correction of restricted socket syndrome, ocular adnexal reconstruction after tumor resection, cicatricial ocular surface diseases and therapy-refractory pterygia. New indications are conjunctival insufficiency after filtrating glaucoma surgery and the combination of mucosa and amniotic membrane grafting for fornix reconstruction. Moreover, different strategies for ex vivo mucous epithelial cell expansion are under discussion. Conclusions: Oral mucosal grafting is a viable option for the replacement of the conjunctiva. Advantages include easily accessibility of grafts in sufficient size even for repeated procedures and a high stability of the grafts. On the other hand, nasal mucosal grafts are superior for some indications due to the lack of goblet cells in the oral mucosa.
\end{abstract}

Copyright $\odot 2013$ S. Karger AG, Basel

\section{KARGER}

(c) 2013 S. Karger AG, Basel

0030-3747/13/0502-0091\$38.00/0

E-Mail karger@karger.com

www.karger.com/ore

\section{Introduction}

A broad variety of techniques, materials and grafts have been used to replace ocular surface tissue [1]. In cases when a conjunctival wound cannot be adapted, secondary healing of the conjunctiva leads to more or less irritating and dysfunctional conjunctival scarring. Conjunctival scarring leads to reduced motility of the globe resulting in diplopia and severe anatomical and functional impairment. Moreover, the loss of the conjunctival secretory functions (goblet cells, accessory tear glands) leads to additional damage of the ocular surface. The choice of the ideal conjunctiva-replacing tissue or material depends on the predominantly lost conjunctival function in the individual situation. In cases when only the conjunctival extension has to be restored, amnion membrane grafting can be a sufficient solution. In conjunctival pathologies with severe goblet cell dysfunction (e.g. ocular pemphigoid), grafts of mucosa containing goblet cells naturally in sufficient number might be more suitable than oral mucosa.

Mucous membrane transplants have been used for over a century in the reconstruction of fornices obliterated by symblepharon formation [1]. The assessment of this technique has changed over time. In recent years, alternative techniques like amnion membrane transplantation have extended the therapeutic options. On the other hand, newer indications like conjunctival insufficiency after trabeculectomy have been added [2].

In the following review, the different indications for oral mucosal grafting are described and discussed in de- 
tail. Moreover, we explain and assess the different surgical procedures of graft removal and placement. The presentation focuses on developments of the last 5 years.

\section{Choice of Graft Material}

The ideal material for fornix reconstruction is a healthy conjunctival or tarsal autograft; however, especially in bilateral disease, there is limited availability.

If conjunctiva or tarsus is not available, a full-thickness oral mucous membrane graft is a simple way to use. Splitthickness mucosal grafts contract more than full-thickness grafts. They are therefore less suitable for fornix reconstruction. But they can serve as an alternative for replacement of the bulbar conjunctiva.

Grafts from the hard palate are the thickest oral mucosal grafts. They contract the least but are more difficult to harvest than other mucosal grafts. So, they should be chosen if sufficient buccal or labial mucosa is not available or when it is desirable to avoid contracture. Oral mucosa does not contain goblet cells and therefore does not support the tear film.

In cases of extreme dry eye situations or severe mucous deficiency syndromes that appear after severe eye burns, nasal mucosal grafts further succeed because they do contain goblet cells that may contribute mucus to the tear film [3]. Nasal septal cartilage contains fewer goblet cells but is useful in the reconstruction of the posterior lamella when the tarsus is lacking; the graft replaces the rigidity of the tarsus, and its attached mucoperichondrium provides a mucosal binding which does not shrink and can be wrapped around the skin of the reconstructed eyelid to form a stable eyelid margin [1] (table 1). In recent years, a combination of different tissue types has been introduced, such as amnion membrane and oral mucosa transplants, in the same eye $[4,5]$. Moreover, a variety of techniques of in vitro expanding oral mucosal epithelium cells have been investigated, e.g. COMET ('cultured oral mucosal epithelial transplantation'). These ongoing studies are part of the growing field of tissue engineering and are excellently summarized in the work of Schrader et al. [6] in 2009. Sotozono et al. [7] treated 46 eyes of 40 patients with complete limbal stem cell deficiency with cultivated oral mucosal epithelial sheet transplantation. Nearly $50 \%$ of those patients with visual loss profited significantly from long-term substantial visual improvement, even patients with end-stage severe ocular surface disorders accompanying severe tear deficiency. Sen et al. [8] characterized the potential of explanted cultured hu-
Table 1. Advantages and disadvantages of different graft materials for conjunctival replacement (modified from Henderson and Collin [1])

\begin{tabular}{|c|c|c|c|}
\hline Graft material & $\begin{array}{l}\text { Oral } \\
\text { mucosa }\end{array}$ & $\begin{array}{l}\text { Nasal } \\
\text { mucosa }\end{array}$ & $\begin{array}{l}\text { Amnion } \\
\text { membrane }\end{array}$ \\
\hline Stability & high & high & low \\
\hline Thickness & thicker & thicker & thinner \\
\hline Color & pink & pink & colorless \\
\hline Goblet cell replacement & no & yes & no \\
\hline Easy accessibility & yes & no & yes \\
\hline Epithelial stem cells & yes & yes & no \\
\hline
\end{tabular}

man oral mucosal epithelial cells to act as autografts for ocular surface reconstruction in patients with bilateral ocular surface disease in order to ameliorate the mucin deficiency state. Inatomi et al. [9] assessed the use of in vitro cultured oral mucosal cell sheets based on amniotic membrane for ocular surface reconstruction in 15 eyes of 12 patients with Stevens-Johnson syndrome or chemical burns, with an average follow-up of 20 months. The corneal surface was stable and transparent, without any major complications in 10 of 15 eyes, and visual acuity improved by more than 2 lines in 10 eyes. Although amniotic membrane-based oral mucosal cell sheets have so far only been applied for corneal surface reconstruction, they might also be suitable for replacement of the conjunctiva.

However, Hirayama et al. [10] compared the clinical results of COMET of substrate-free sheets with those of COMET of amniotic membrane-based sheet on the corneal surface of patients with chemical injury and pseudoocular cicatricial pemphigoid. The authors found a better midterm clinical outcome after COMET with a substratefree cell sheet and concluded that results with amniotic membrane grafts in the presence of chronic inflammatory ocular surface disease are less satisfactory.

Other potential donor sites such as rectal mucosa or vaginal mucosa also contain a high number of goblet cells. Anyhow, when regarding the additional time to harvest, risks for donor site complications and donor site pain, ophthalmologists and patients may hesitate to use these tissues.

\section{Indications}

\section{Restricted Socket Syndrome/Postenucleation Socket} Syndrome

Contracted sockets are commonly seen many years after enucleation. Most surgeons agree that grafts in the
Mai/Bertelmann 

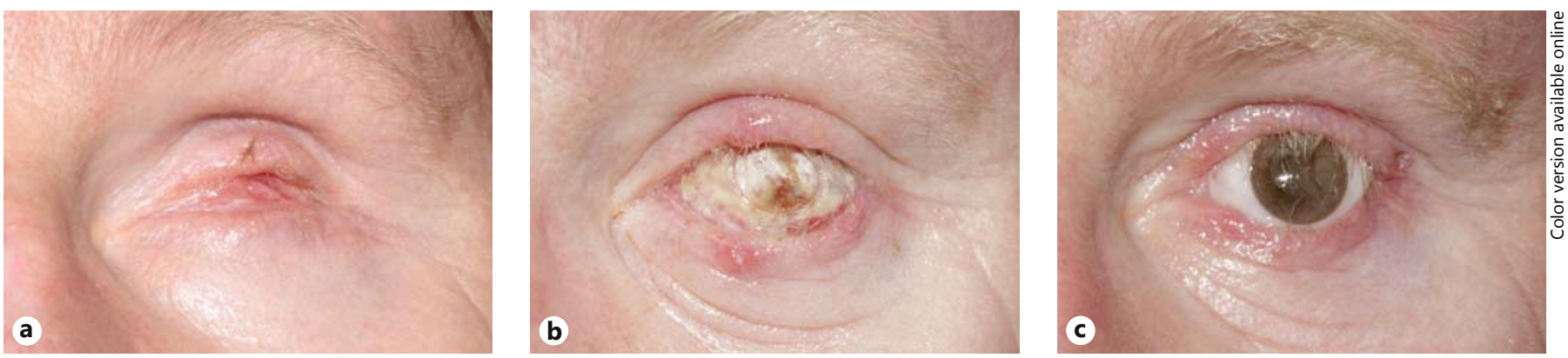

Fig. 1. a Victim of acid burns of both eyes 20 years ago. Appearance following enucleation of the left eye. Contracted socket with completely scarred fornices. b Clinical finding 1 week after reconstruc- tion of upper and lower fornix with dermis fat transplantation from the gluteal region and buccal mucosal graft, conformer (Illig shell) in place. c View 3 months postoperatively with the prosthesis in place. socket contract rapidly and that a pad, stent or prosthesis needs to be kept in the socket at all times for many weeks in order to prevent recurrence of socket contracture. Mustardé referred to the contracture of the socket as 'malignant', so relentless is the process [11].

Atrophied orbital fat can be replaced by dermis fat grafts from the gluteal region. Oral or buccal mucosa is the tissue of choice to reconstruct the conjunctival part of a contracted socket. It is also a suitable tissue for reconstructing the conjunctiva of the globe in patients with functioning eyes, as the graft can be cut fairly thin and is pliable.

The use of other tissues, including split skin, palatal mucosa, labial mucosa and foreskin, has been described by various authors. Some have favored split skin because it is in plentiful supply, but most dislike the offensive discharge that occurs when both skin and mucosa are present in the orbit. Those who support the use of split skin maintain that this problem lessens with time.

The use of palatal mucosa and nasal septal mucosa results in less contraction, but there is less material available for harvesting and the grafts are less pliable. The fact that buccal mucosa can be harvested repeatedly in generous amounts with no morbidity makes it the tissue of choice in case of restricted socket syndrome (fig. 1a, b).

Recently Yoon et al. [12] documented the benefit of oral mucosa transplantation for exposed hydroxyl apatite orbital implants. Ten out of 13 exposed hydroxyl apatite balls could be successfully treated by this technique in this study.

Conjunctival Replacement following Tumor Resection

Eyelid malignancies have a varied pathology including basal cell carcinoma, squamous cell carcinoma, malignant melanoma, sebaceous gland carcinoma and other rare tumors like hemangiopericytoma. Resection of eyelid malignancies leads to complex reconstructive problems due to the functional and aesthetic importance of an eyelid.

Eyelid defects following tumor excision can be fixed by various techniques including primary closure, healing by secondary intention, flaps or grafts. Selecting the appropriate technique is determined by assessing the size of the defect, the health of the surrounding residual structures, the visual acuity of the contralateral eye, the age and medical health of the patient, and the surgeon's own expertise. When bulbar conjunctiva is involved in the area of excision, oral mucosa might be required to cover the defect.

Half of our patients who had undergone mucosal graft surgery on the ocular surface during the last 5 years had eyelid malignancies. Even though basal cell carcinoma is the most common tumor of the eyelid, it is mostly limited to the eyelid without entering the bulbar conjunctiva. The latter, however, is often the case with squamous cell carcinoma, which in our patients was the most malignant tumor of the eyelid requiring reconstruction of the bulbar conjunctiva (fig. 2a-c). A more infrequently occurring malignancy of the conjunctiva is malignant melanoma. Treatment of this tumor often requires mucosal grafting due to large required resection areas (fig. $3 \mathrm{a}-\mathrm{d}$ ).

However, a mucosal graft was included in the reconstruction strategy only in 1 case (of 6) in a study by De Sousa et al. [13] on total upper and lower eyelid reconstruction.

\section{Cicatricial Ocular Surface Diseases}

Cicatricial ocular surface diseases such as ocular cicatricial pemphigoid, Stevens-Johnson syndrome, toxic epidermal necrolysis, and chemical burns encompass a 

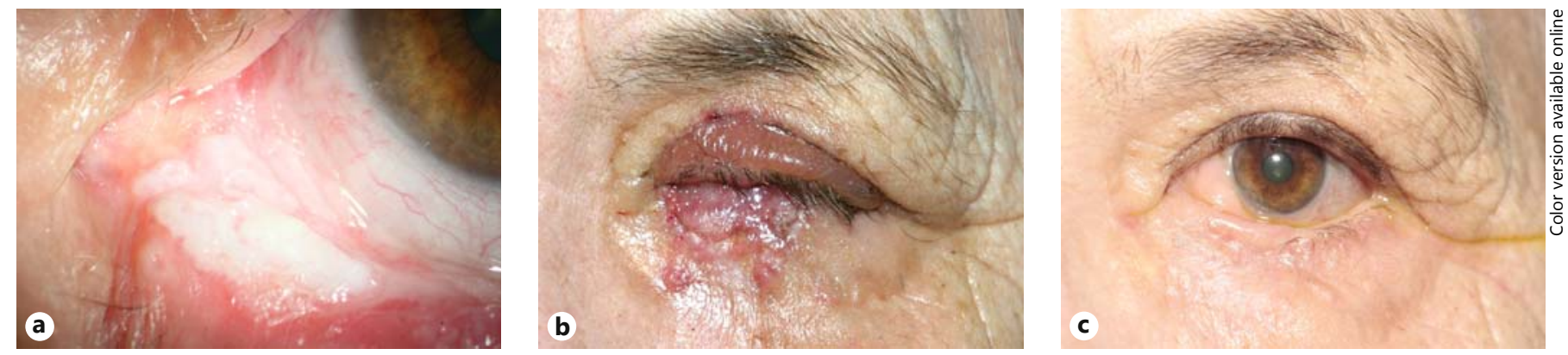

Fig. 2. a Squamous cell carcinoma of the eyelid and the tarsal conjunctiva. b Excision and eyelid reconstruction with a Hughes tarsoconjunctival flap, 1 week postoperatively. c Clinical result 3 months postoperatively.

Fig. 3. a, b Circumscribed malignant melanoma of the conjunctiva in a large area of melanocytosis covering the whole circumference. c, d Reconstruction (after excision and cryotherapy of cornea) using a Hughes tarsoconjunctival flap and free buccal mucosal grafting for bulbar conjunctiva; 6 months postoperatively, result showing adequate function.
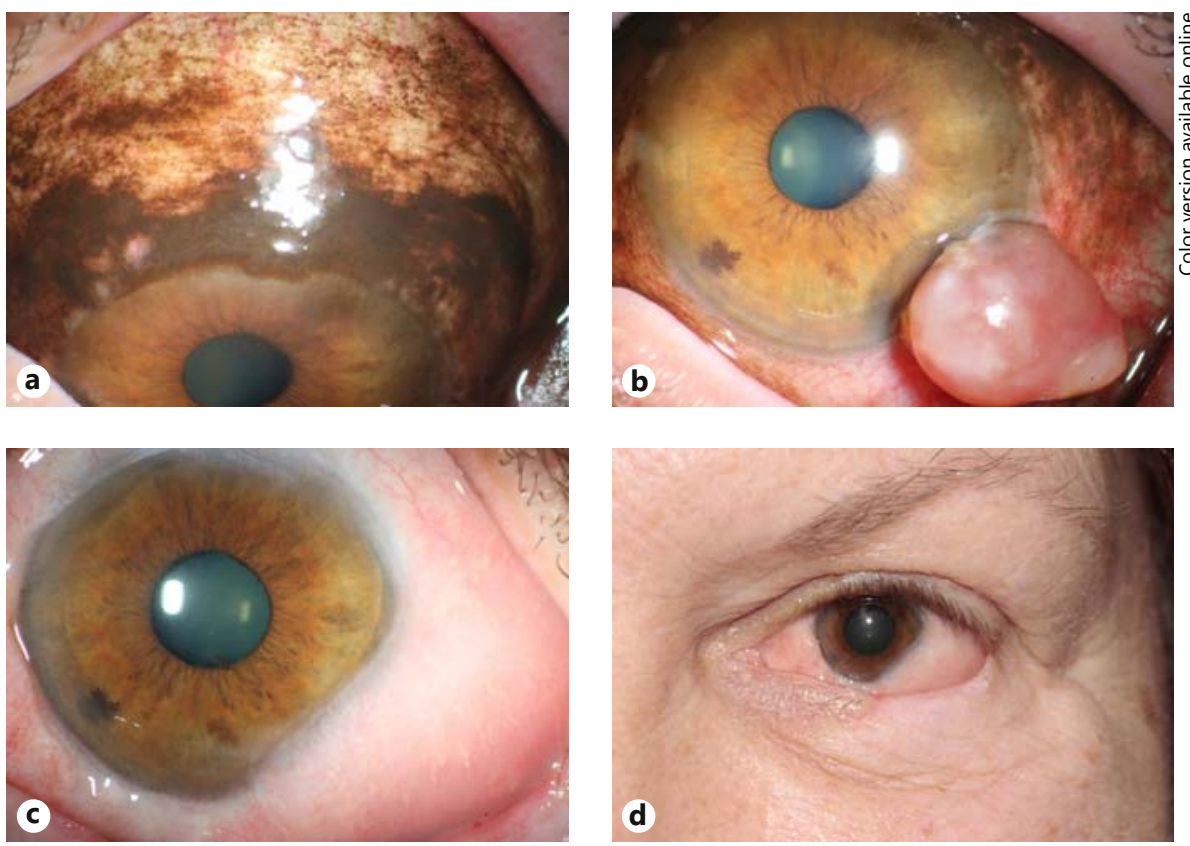

myriad of ocular complications leading to significant visual morbidity. Scarring in the lid margin, the tarsus, and the fornix may lead to keratinization, trichiasis, distichiasis, and entropion. These lid margin and lash abnormalities can cause chronic blink-related microtrauma to the ocular surface and have recently been shown to be significantly correlated with persistent ocular surface inflammation and subsequent corneal blindness. These cicatricial complications can also cause incomplete blinking or closure and interfere with the Bell phenomenon, resulting in unwanted exposure of the ocular surface. Such an exposure problem may occur not only during the day when the eye is open, but also during sleep, resulting in lagophthalmos. Cumulative insults from both blink-related microtrauma and chronic exposure further deteriorate the ocular surface and contribute to higher failure rates when ocular surface reconstructive procedures are contemplated [14].

For these two pathogenic elements, that is, blink-related microtrauma and incomplete closure, large-diameter contact lenses and special scleral lenses do protect the cornea to a certain extent. However, lens fitting can be a problem in eyes with significant symblepharon, and compliance is particularly poor in these cicatricial diseases. The long-term use of bandage contact lenses and scleral lenses is not without complications, especially in eyes 

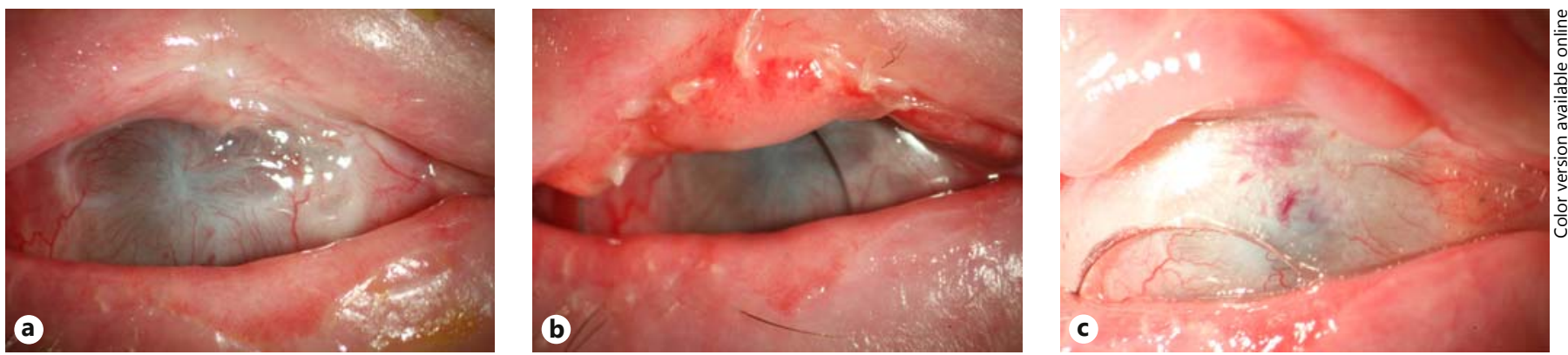

Fig. 4. a Victim of burn by pyrotechnic article: massive corneal vascularization and firm symblepharon of the upper fornix. b One week postoperatively, the conjunctival vascularized graft in the up-

per fornix. c Clinical result after reconstruction of the upper fornix using buccal mucous grafting, the plastic conformer in place (upper lid elevated by examiner), corneal vascularization slightly reduced.

with sicca [15]. Thus, a frequently favored option is a surgical attempt to improve cicatricial eye diseases if the underlying pathophysiological process is controlled.

Oral mucosal graft is commonly used during oculoplastic surgeries to replace the ocular (corneal and conjunctival) surface as well as for reconstruction of the eyelid, the fornix [16] and the socket [1,17]. For reconstruction of the lid margin, oral mucosal graft can correct trichiasis and entropion. Recently, Iyer et al. [18] used mucous membrane grafting for correcting lid margin keratinization in Stevens-Johnson syndrome to reduce ocular surface inflammation and to improve patient comfort and visual acuity. However, their study excluded severe cases with extensive symblepharon, entropion, ocular surface cicatrization, and severe dry eye. In our experience, oral mucosal graft can be used not only for correcting distichiasis causing blink-related microtrauma, but also for incomplete closures that are frequently found in severe cicatricial ocular surface diseases (fig. $4 \mathrm{a}-\mathrm{c}$ ).

In 2008, Kheirkhah et al. [16] proposed a grading system for symblepharon and a grade-adjusted step therapy (amniotic membrane transplantation, plus anchoring sutures, plus conjunctival autograft and/or oral mucosal transplantation). Furthermore, intraoperative additional use of topical mitomycin $\mathrm{C}$ may be useful (mitomycin C $0.04 \%$ soaked sponge for $3-5 \mathrm{~min}$ ). For this strategy, the authors reported an overall success rate of $85 \%$ for one procedure after 25 months.

In the view of Kim et al. [3] in 2010, autologous nasal mucosa is much more suitable than oral mucosal grafting for the ocular surface reconstruction in cicatricial ocular surface diseases. Although the published experiences are based on only 6 patients in this study, extensive historical investigations into nasal mucosa grafting support these findings [19]. Even though a great amount of goblet cells is already lost during surgery, mucin production increased after surgery, and the persistence of goblet cells on the ocular surface could be shown for up to 10 years. The authors' histologic and immunohistochemical analyses show PAS staining and MUC5AC immunostaining as a marker for mucin-containing goblet cells only in nasal mucosa and not in oral mucosal epithelium. However, nasal mucosa is derived from the respiratory tract and may produce mucins differing from those of the ocular surface as produced by epithelial cells of the conjunctiva [19].

In contrast, $\mathrm{Fu}$ et al. [20] reported good experience with the use of oral mucosa graft in cicatricial ocular surface diseases based on the evaluation of 22 eyes of 19 patients. Foreign body sensation, photophobia, pain, burning, tearing and discharge improved in $77.3 \%$ of the eyes.

An additional indication for buccal mucous membrane grafting is complicated lacrimal duct obstruction which was shown by Tao et al. [21] in 2010. The authors published the results of 11 patients after failed previous lacrimal surgery treated with mucous membrane graftassisted dacryocystorhinostomy.

\section{Treatment-Refractory Pterygium}

Pterygium is a commonly encountered problem and, although frequently asymptomatic, may cause symptoms due to irritation, astigmatism, and reduced visual acuity which necessitate its removal. Current first-line surgical procedures include simple excision leaving bare sclera; excision and conjunctival closure by primary anastomosis or conjunctival grafts, and excision combined with $\beta$-irradiation, mitomycin, or thiotepa. The commonest complication is recurrence of the pterygium and the most severe complication is scleral melt and eyeball perfora- 

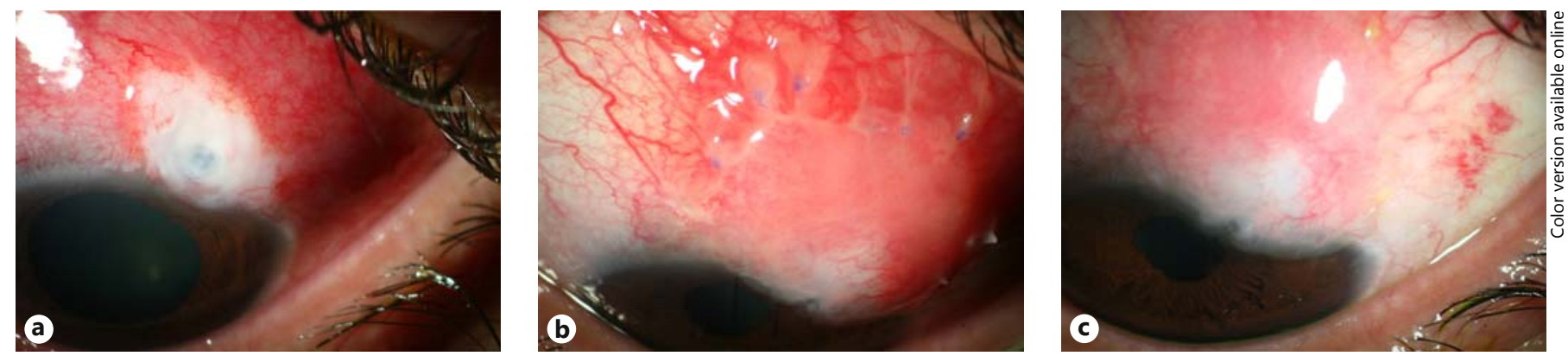

Fig. 5. a Thin, avascular bleb with positive Seidel test. Reconstruction of insufficient bleb with amniotic membrane and autologous free conjunctival graft has been frustrating. Intraocular pressure is $3 \mathrm{~mm} \mathrm{Hg}$. b Buccal mucous membrane reconstruction of trabecu- lectomy bleb, looking pink, vascularized and viable 1 week postoperatively; intraocular pressure is $10 \mathrm{~mm} \mathrm{Hg}$. c Clinical result 3 months postoperatively showing vascularized, conveyed bleb in adequate function, intraocular pressure is $10 \mathrm{~mm} \mathrm{Hg}$. tion. The reported frequency of recurrence for primary pterygium varies widely for different procedures, ranging from $24-67 \%$ of cases after simple excision by the bare sclera technique, to $0-47 \%$ of cases after excision combined with conjunctival autografting or adjuvant therapy [22]. Recurrences may grow aggressively, rapidly reaching and even overtaking the size of the original pterygium. Many different techniques have been suggested for dealing with recurrent pterygia, including excision combined with lamellar keratoplasty, autologous conjunctival grafts, $\beta$-irradiation, and antimitotics such as mitomycin C, 5-fluorouracil, and thiotepa, resulting in further recurrence of the pterygium in up to $30 \%$ of such cases. Even if today cases of repeated recurrence are rare, they may develop severe conjunctival scarring and shortening, resulting in insufficient conjunctiva to perform further grafting and lid surgery, and restricted eye movements. Patients from tropical countries may also have trachomatous scarring and xerosis, which reduces the amount of conjunctiva available for autologous grafting. Under these circumstances, mucous membrane must be obtained from other sites.

The advantages attributed to the use of split-thickness buccal mucous membrane compared to full-thickness grafts are a better cosmetic appearance and possibly less contraction.

We treated 3 patients who had repeated surgery for pterygium and who finally benefitted from buccal mucosal graft transplantation with good results.

\section{Conjunctival Insufficiency after Filtration Surgery}

Trabeculectomy is the most commonly performed surgery to lower intraocular pressure. Although it is a safe and effective procedure, it is not always without compli- cations. One of the more concerning of these is late-onset bleb leak caused primarily by inappropriate handling of the conjunctiva including the use of cytotoxic agents. Such leaks can lead to hypotony, choroidal effusion, corneal decompensation, maculopathy, blebitis, and endophthalmitis.

In order to avoid these complications, bleb leaks should be repaired in a timely manner. A wide variety of nonincisional strategies such as aqueous suppressant therapy or oversized badge contact lenses have been used, with limited long-term efficacy. Often, these leaks require surgical repair usually involving reconstruction of the bleb using local conjunctiva, mobilized through either an advancement or pedicle flap. If this is not possible, either a free autologous conjunctival graft or amniotic membrane transplantation can be used [23]; both with variable results. In complicated cases, these attempts to control the leak may be without success.

If this is the case, an alternative to both of these tissues for bleb reconstruction might be buccal mucous membrane grafting. It has first been described by Rootman et al. [2] in 2010. Figure 5 shows an example of the clinical course of this technique. Surgically, the principles are the same as with conjunctival transplantation, in that buccal transplant involves the harvest of autologous mucous membrane from a distant site with transplantation in a tension-free manner to the site of the excised bleb. But it is important to note that buccal membrane tissue differs from conjunctiva in that it heals differently. By establishing vascular perfusion, it becomes congested. It is also thicker than the conjunctiva and forms a lower bleb. An additional advantage is saving the conjunctiva for further glaucoma surgery if indicated.
Mai/Bertelmann 


\section{Surgical Procedures}

\section{Harvest of Full-Thickness Buccal Mucosal Graft}

The surgery is carried out under general anesthesia with the endotracheal tube secured to one side of the mouth so as to allow adequate exposure of the oral mucosa on the other side and using a surgical eye loupe in order that the detailed excision described below could be executed precisely. After the defect occurring in the conjunctiva is estimated, the attention is directed to the buccal oral mucosa, were submucosal injection of local anesthetic is given. It is important to adjust the position of harvesting to avoid the opening of the parotid duct, usually opposite the second upper molar. A free mucosal graft a little bigger than the estimated defect is harvested by demarcating the border with a superblade followed by scissor dissection. The occurring wound is then closed by $5 \times 0$ Vicryl suture. The excess fat is cleared from the stromal surface of the graft with curved Wescott scissors.

\section{Reconstruction of the Fornix}

If marked contraction of the conjunctiva of the socket is established, extra mucosa must be inserted. Reconstruction is performed under general anesthesia. The lower fornix is reformed and scar tissue is dissected by a transverse incision through the contracted conjunctiva of the lower fornix. After the incision is deepened with scissors to expose the periosteum of the inferior orbital rim, enough oral mucosal graft is inserted to create a new fornix without tension. Deepening of the reconstructed fornix should be supported by fixation to the periosteum of the orbital rim by sutures tied over strips on the skin.

The conjunctiva of the upper fornix is incised transversely close to the upper border of the tarsal plate and reflected from the upper lid retractors. Then the dissection is continued beyond the uppermost extent of the fornix. The graft is inserted and supported with a conformer formed by one half of a silicone tube sutured in place with sutures which pass through the full lid thickness.

\section{Reconstruction of Filtering Bleb}

A lid speculum is inserted and 7/0 silk sutures placed through the clear cornea for retraction and exposure of the affected bleb. Xylocaine $1 \%$ is injected subconjunctivally surrounding the atrophic bleb. A blade is used to perform a shallow keratectomy anterior to the bleb edge. The bleb is removed from the sclera. The edge of the residual flap is sutured edge to edge with the buccal mucous graft with a resorbable $9 / 0$ suture.

\section{Postoperative Management}

Topical antibiotics and steroids are administered 4 times a day for approximately 1 month. The mucous graft tissue is initially white and avascular but after 1 week it is getting pink, vascularized and viable looking. Over time, the vascularity and pink coloration fades somewhat (fig. 2c). Patients are seen once a week during the first postoperative month by an ophthalmologist in order to avoid formation of postoperative symblepharon. In case of fornix reconstruction the performer (Illig shell) should stay in the newly created fornix for at least 1 up to 3 months. Changing and cleaning of the performer is done once a week.

\section{Conclusions}

Among various alternative and in part newer techniques, oral mucosal grafting is still an up-to-date suitable procedure for the replacement of the conjunctiva. Advantages include easily accessibility of grafts in sufficient size even for repeated procedures, fast and cheap grafting in contrast to ex vivo methods and a high stability of the grafts. For some indications, nasal mucosal grafts might be superior to oral mucosal transplants due to the lack of goblet cells in the oral mucosa. The clinical experience of the benefit of ex vivo propagated mucosal epithelial cells for treatment of ocular surface disease and limbal stem cell insufficiency will probably markedly increase in the near future.

\section{References}

1 Henderson HWA, Collin JRO: Mucous membrane grafting; in Geerling G, Brewitt H (eds): Surgery for the Dry Eye. Dev Ophthalmol. Basel, Karger 2008, vol 41, pp 230-242.

2 Rootman DB, Kumar NL, Rootman DS, Trope GE: Buccal mucous membrane for the reconstruction of complicated leaking trabeculectomy blebs. J Glaucoma 2010;19:270-274.
3 Kim JH, Chun YS, Lee SH, Mun SK, Jung HS, Lee SH, Son Y, Kim JC: Ocular surface reconstruction with autologous nasal mucosa in cicatricial ocular surface disease. Am J Ophthalmol 2010;149:45-53.

4 Liu J, Sheha H, Fu Y, Giegengack M, Tseng SCG: Oral mucosal graft with amniotic membrane transplantation for total limbal stem cell deficiency. Am J Ophthalmol 2011;152: 739-747.

5 Kheirkhah A, Ghaffari R, Kaghazkanani R, Hashemi H, Behrouz MJ, Raju VK: A combined approach of amniotic membrane and oral mucosa transplantation for fornix reconstruction in severe symblepharon. Cornea 2013;32:155-160. 
6 Schrader S, Notara M, Beaconsfield M, Tuft SJ, Daniels JT, Geerling G: Tissue engineering for conjunctival reconstruction: established methods and future outlooks. Curr Eye Res 2009;34:913-924.

7 Sotozono C, Inatomi T, Nakamura T, Koizumi N, Yokoi N, Ueta M, Matsuyama K, Miyakoda K, Kaneda H, Fukushima M, Kinoshita S: Visual improvement after cultivated oral mucosal epithelial transplantation. Ophthalmology 2013;120:193-200.

8 Sen S, Sharma S, Gupta A, Gupta N, Singh H, Roychoudhury A, Mohanty S, Sen S, Nag TC, Tandon R: Molecular characterization of explant cultured human oral mucosal epithelial cells. Invest Ophthalmol Vis Sci 2011;52: 9548-9554.

9 Inatomi T, Nakamura T, Koizumi N, Sotozono C, Yokoi N, Kinoshita S: Midterm results on ocular surface reconstruction using cultivated autologous oral mucosal epithelial transplantation. Am J Ophthalmol 2006;141: 267-275.

10 Hirayama M, Satake Y, Higa K, Yamaguchi T, Shimazaki J: Transplantation of cultivated oral mucosal epithelium prepared in fibrincoated culture dishes. Invest Ophthalmol Vis Sci 2012;53:1602-1609.
11 Mourits MP: A short history of contemporary oculoplastic surgery: excerpts from the Mustardé lecture 2011. Orbit 2012;31:270-273.

12 Yoon KC, Yang Y, Jeong IY, Kook MS: Buccal mucosal graft for hydroxyapatite orbital implant exposure. Jpn J Ophthalmol 2011;55: 318-320.

13 De Sousa JL, Leibovitch I, Malhotra R, O’Donnell B, Sullivan T, Selva D: Techniques and outcomes of total upper and lower eyelid reconstruction. Arch Ophthalmol 2007;125: 1601-1609.

14 Liang L, Sheha H, Tseng SC: Long-term outcomes of keratolimbal allograft for total limbal stem cell deficiency using combined immunosuppressive agents and correction of ocular surface deficits. Arch Ophthalmol 2009;127: 1428-1434.

15 Sindt CW, Longmuir RA: Contact lens strategies for the patient with dry eye. Ocul Surf 2007;5:294-307.

16 Kheirkhah A, Blanco G, Casas V, Hayashida Y, Raju VK, Tseng SC: Surgical strategies for fornix reconstruction based on symblepharon severity. Am J Ophthalmol 2008;146:266275.

17 Lopes N, Castel G, Andres R, Lisboa M, Castela R, Loureiro R: Reconstruction of anophthalmic socket. J Fr Ophthalmol 2011;34:608614.
18 Iyer G, Pillai VS, Srinivasan B, Guruswami S, Padmanabhan P: Mucous membrane grafting for lid margin keratinization in Stevens Johnson syndrome (SJS). Cornea 2010;29:146151.

19 Wenkel H, Rummelt V, Naumann GOH: Long term results after autologous nasal mucosal transplantation in severe mucus deficiency syndromes. Br J Ophthalmol 2000;84: 279-284.

20 Fu Y, Liu J, Tseng SCG: Oral mucosal graft to correct lid margin pathologic features in cicatricial ocular surface diseases. Am J Ophthalmol 2011;152:600-608.

21 Tao JP, Luippens D, McCord CD: Buccal mucous membrane graft-assisted lacrimal drainage surgery. Ophthalmol Plast Reconstr Surg 2010;26:39-41.

22 Hirst LW: Recurrence and complications after 1,000 surgeries using pterygium extended removal followed by extended conjunctival transplant. Ophthalmology 2012;119:22052210.

23 Rauscher FM, Barton K, Budenz DL: Longterm outcomes of amniotic membrane transplantation for repair of leaking glaucoma filtering blebs. Am J Ophthalmol 2007;5:10521054. 